\section{Enfermedad de Creutzfeldt-Jakob: experiencia de 5 años en un hospital terciario de Chile}

\author{
SERGIO LEGUA KOC ${ }^{1}$, PAULA CASTILLO TORRES ${ }^{2}$, \\ ALFONSO LEÓN MANTERO ${ }^{3,4}$, MANUEL ALVARADO PASTENES ${ }^{3,4}$, \\ GLADYS GODOY REYES ${ }^{3,4}$, DAVID SÁEZ MÉNDEZ ${ }^{3,4}$, \\ JOSÉ LUIS BUCAREY TAPIA ${ }^{5}$
}

\section{Creutzfeldt-Jakob disease, experience in 17 patients}

Background: Creutzfeldt-Jakob disease (CJD) is a prion affection that typically produces a rapidly progressive dementia with different neurologic and extra-neurologic manifestations. Aim: To characterize clinical, imaging and electroencephalography findings in patients with a probable CJD. Patients and Methods: A case series study of patients admitted in the Neurology department at a public hospital, between 2014 and 2019. Demographic, clinical, imaging, and electroencephalographic data of patients with probable CJD were analyzed. Results: Seventeen patients aged $63 \pm 11$ years (53\% women) with a probable CJD were gathered. The incidence was 4.7 cases/year per million inhabitants. Twenty four percent of patients had a family history of CJD. The median time between the onset of symptoms and the hospital admission was three months with a survival of four months. The most common clinical manifestations were an amnesic syndrome in $88 \%$, myoclonus in $76 \%$, frontal syndrome and ataxia in $71 \%$. Brain MRI was abnormal in all patients. The preponderant finding was the involvement of the caudate nucleus in $82 \%$ of cases. In the EEG, $94 \%$ of patients had abnormalities. All had a theta-delta slowing as a base rhythm. The pseudo-periodic pattern was observed in the 29\% and status epilepticus in $18 \%$. Conclusions: In this group of patients we observed the heterogeneity of the clinical manifestations of the disease, the frequent imaging and electroencephalographic alterations and the short evolution time leading to death.

(Rev Med Chile 2021; 149: 1285-1291)

Key words: Dementia; Creutzfeldt-Jakob Syndrome; Prion Diseases; Status Epilepticus.

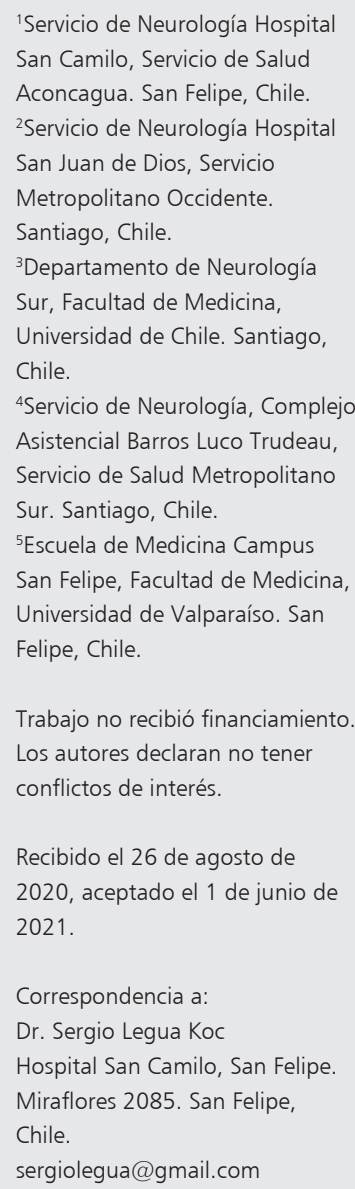

L as enfermedades priónicas son patologías neurodegenerativas de rápido avance con desenlace fatal, provocadas por el mal plegamiento de la proteína priónica, la cual, al contactarse con otras proteínas normales, las transforma en patológicas, aumentando exponencialmente su número en el tejido cerebral ${ }^{1}$.

La enfermedad priónica más descrita es la enfermedad de Creutzfeldt-Jakob (ECJ), la que puede clasificarse en esporádica (85\%), familiar $(10 \%)$, iatrogénica y variante ${ }^{2}$. El sello fisiopatológico de la enfermedad es el mal plegamiento de la proteína priónica celular $\left(\mathrm{PrP}^{\mathrm{c}}\right)$ que normalmente presenta una conformación de hélices alfa, en una donde predominan las láminas beta, llamada proteína priónica scrapie $\left(\mathrm{PrP}^{\mathrm{sc}}\right)$, menos soluble y susceptible de proteólisis ${ }^{2,3}$. Posteriormente se deposita en diferentes zonas del encéfalo, prin- 
cipalmente en corteza cerebral, ganglios basales, tálamo y cerebelo, con posterior activación de la microglía, neuroinflamación y finalmente neurodegeneración y espongiosis ${ }^{2,4}$. La $\mathrm{PrP}^{\mathrm{c}}$ es codificada por el gen PRNP en el cromosoma 20, cuyas mutaciones dan origen a las formas familiares de la enfermedad y, por otra parte, presenta un polimorfismo en el codón 129, que puede codificar el aminoácido valina $(\mathrm{V})$ o metionina $(\mathrm{M})$. Basado en este polimorfismo, se ha agrupado la enfermedad en 6 diferentes genotipos (MM1, MV1, VV2, MV2, VV1 y MM2) que se condicen con ciertas características clínicas y anatomopatológicas ${ }^{2,3,4,5}$.

La incidencia anual mundial de la ECJ esporádica es de 1-2 casos por millón de habitantes ${ }^{2,4,6}, y$ según series nacionales, la incidencia en Chile sería mayor, con 3,5 casos por millón de habitantes ${ }^{7,8}$. Los criterios diagnósticos de la ECJ esporádica han cambiado a través de la historia, según la existencia de exámenes paraclínicos que apoyan la sospecha, siendo el diagnóstico definitivo neuropatológico. Los criterios inicialmente se basaban en la clínica y electroencefalograma (EEG), luego se añadieron la proteína 14-3-3 en líquido cefalorraquídeo (LCR) y la resonancia magnética (RM) de cerebro, hasta la actualidad, en que se han identificado diferentes mutaciones del gen PRNP y polimorfismos en el codón $129^{5} \mathrm{y}$, más recientemente, la técnica fluorimétrica real-time quaking-induced conversion (RT-QuIC) que detecta pequeñas cantidades de proteína priónica anormal en LCR u otros tejidos ${ }^{9}$. Los criterios diagnósticos actuales para ECJ probable requieren la presencia de un síndrome demencial rápidamente progresivo asociado a dos o más de los siguientes signos clínicos: mioclonías, alteración visual/cerebelosa, síntomas o signos piramidales o extrapiramidales y mutismo akinético, $y$, al menos, un examen paraclínico alterado (RM, EEG, proteína 14-3-3 o RT-QuIC) ${ }^{10,11}$.

A continuación, presentamos un estudio de las características clínicas, imagenológicas y electroencefalográficas de 17 pacientes con diagnóstico de ECJ probable.

\section{Pacientes y Métodos}

Estudio de una serie de casos donde se revisaron fichas clínicas de 53 pacientes hospitalizados en el Servicio de Neurología del Hospital Barros Luco Trudeau (HBLT) de Santiago de Chile, con el diagnóstico de ingreso o egreso de "síndrome demencial en estudio" o "síndrome demencial rápidamente progresivo", en el período comprendido entre enero de 2014 y enero de 2019. Luego de la revisión de sus fichas clínicas, se seleccionaron 17 pacientes que cumplían los criterios diagnósticos para ECJ probable. Se obtuvieron datos de edad al diagnóstico, sexo, factores de riesgo cardiovascular, antecedente de familiar en primer grado afectado por ECJ, además de las fechas de inicio de los síntomas, de hospitalización para estudio y de fallecimiento, con lo cual se obtuvieron los tiempos desde el inicio de síntomas a la hospitalización y tiempo de sobrevida. Se obtuvo información respecto de síntomas y signos al momento del diagnóstico, agrupándose en síndromes: frontal y sus tres divisiones anátomo-funcionales (dorsolateral/disejecutivo, medial/apático y orbitofrontal/desinhibitorio), amnésico, alucinatorio, afásico/mutismo akinético, alteraciones visuales, piramidal, extrapiramidal, atáxico-cerebeloso, mioclónico, convulsivo y status epiléptico (focal o generalizado). Todos los pacientes fueron sometidos a estudio con RM de cerebro de 1.5 tesla y EEG estándar. En todos los casos se obtuvieron secuencias de Diffusion-weighted Imaging (DWI), Apparent Diffusion Coefficient (ADC) y fluid attenuated inversión recovery (FLAIR). Los hallazgos patológicos en la RM, dados por la presencia de hiperintensidad en secuencias de DWI (con correlato en ADC) y FLAIR, se agruparon según topografía en: corteza frontal, parietal, temporal, occipital e insular, núcleo caudado, tálamo y putamen, y lateralidad (derecha o izquierda). Los hallazgos electroencefalográficos se agruparon según el patrón predominante encontrado: normal, lentitud theta-delta generalizada, actividad epileptiforme interictal (puntas, puntas lentas o punta-onda lenta focal o generalizada), descargas epileptiformes lateralizadas periódicas (periodic lateralized epileptiform discharges, PLEDs), patrón pseudoperiódico (ondas bi o trifásicas de duración 100-300 milisegundos, a 0,5-2 Hertz) y status epiléptico convulsivo (SEC) focal o generalizado. Tanto para RM como para EEG, se cuantificó el tiempo en meses transcurrido desde el inicio de los síntomas a la realización del examen. Todos los pacientes fueron sometidos a exámenes de diagnóstico diferencial que incluyeron laboratorio general, nivel vitamina B12, serología sífilis, VIH, TC de tórax-abdomen-pelvis, estudio de LCR y 
en un caso panel de encefalitis autoinmune, los cuales fueron negativos en la serie de pacientes presentada. En HBLT no se cuenta con estudio de proteína 14-3-3, por lo que no se realizó. Los datos fueron recolectados de manera anonimizada en una planilla Excel y analizados con el software STATA v16. Se presentan como promedio y desviación estándar o mediana, Q1 y Q3, según su distribución.

Este estudio fue evaluado y aprobado por el Comité de Ética del Servicio de Salud Metropolitano Sur.

\section{Resultados}

\section{Antecedentes generales}

De los 17 pacientes estudiados, el promedio de edad al momento del diagnóstico fue de 62,6 años (DS 10,5). Hubo dos pacientes menores de 50 años (45 y 46 años). Se encontró una leve preponderancia femenina (53\%). El 23,5\% de los enfermos tenía antecedente familiar de ECJ. La mediana del tiempo entre el inicio de los síntomas y la hospitalización fue de 3 meses (Q1: 2, Q3: 5). La mediana de tiempo de sobrevida desde el inicio de los síntomas hasta el fallecimiento fue de 4 meses (Q1: 4, Q3: 11). La edad promedio de fallecimiento fue de 63 años (DS 10,6) (Tabla 1).

\section{Presentación clínica}

Los hallazgos clínicos más frecuentes al momento del diagnóstico fueron el síndrome amnésico (88\%), en segundo lugar, el síndrome mioclónico $(76 \%)$ y en tercer lugar tanto el síndrome atáxico-cerebeloso como el síndrome frontal $(70,6 \%)$. Cabe destacar que $29,4 \%$ se presentó con un síndrome convulsivo y 17, $6 \%$ presentó un SEC (Tabla 2).

\section{Resonancia magnética (RM)}

La mediana de tiempo desde el inicio del cuadro hasta que se realizó la RM fue de 2 meses (Q1: 2, Q3: 5). Todos los pacientes presentaron alteraciones. El compromiso del caudado fue el hallazgo más frecuente $(82,4 \%)$, seguido de la corteza frontal $(70,6 \%)$ y en tercer lugar la corteza parietal y el tálamo (52,9\% cada uno) (Tabla 3). En cuanto a la lateralidad, en general se aprecia una simetría de las topografías afectadas (Figura 1).
Tabla 1. Resumen de antecedentes generales

\begin{tabular}{lll}
\hline $\begin{array}{l}\text { Edad promedio al } \\
\text { diagnóstico }\end{array}$ & $62,6( \pm 10,5)$ años & \\
$\begin{array}{l}\text { Distribución por } \\
\text { rango etario }\end{array}$ & Años & $\mathrm{n}(\%)$ \\
& $50-60$ & $2(11,8)$ \\
& $61-70$ & $4(23,5)$ \\
& $71-80$ & $9(53)$ \\
& $>80$ & 0 \\
Sexo & Mujeres & $2(11,8)$ \\
& $\mathrm{n}=9(52,9 \%)$ & $\mathrm{n}=8(47,1 \%)$ \\
$\begin{array}{l}\text { Factores de riesgo } \\
\text { cardiovascular }\end{array}$ & Hipertensión arterial & $\mathrm{n}=9(53 \%)$ \\
& Dislipidemia & $\mathrm{n}=5(29,4 \%)$ \\
& Diabetes mellitus & $\mathrm{n}=3(17,6 \%)$ \\
& Tabaquismo & $\mathrm{n}=2(11,8 \%)$ \\
Antecedente & $\mathrm{n}=4(23,5 \%)$ & \\
familiar de ECJ & & \\
$\begin{array}{l}\text { Edad promedio de } \\
\text { fallecimiento }\end{array}$ & $63( \pm 10,6)$ años & \\
\hline
\end{tabular}

Tabla 2. Síndromes clínicos al momento del diagnóstico

\begin{tabular}{|ll|}
\hline Síndrome & n (\%) \\
\hline Frontal (total) & $12(70,6)$ \\
- Orbitofrontal & $7(41,3)$ \\
- Medial & $4(23,5)$ \\
- Dorsolateral & $10(58,8)$ \\
\hline Amnésico & $15(88,2)$ \\
\hline Alucinatorio & $6(35,3)$ \\
Afásico/mutismo akinético & $7(41,2)$ \\
\hline Alteraciones visuales & $2(11,8)$ \\
\hline Piramidal & $6(35,3)$ \\
\hline Extrapiramidal & $2(11,8)$ \\
Atáxico-cerebeloso & $12(70,6)$ \\
\hline Mioclónico & $13(76)$ \\
\hline Convulsivo & Inicio generalizado: $4(23,5)$ \\
& Inicio focal: $1(5,9)$ \\
& Total: $5(29,4)$ \\
\hline Status convulsivo & Generalizado: $2(11,8)$ \\
& Focal: $1(5,9)$ \\
& Total: $3(17,6)$ \\
\hline
\end{tabular}


Tabla 3. Hallazgos de RM cerebro (hiperintensidad DWI/FLAIR con correlato en ADC) al momento del diagnóstico

\begin{tabular}{|lc|}
\hline Topografía & n (\%) \\
\hline Corteza frontal & $12(70,6)$ \\
\hline Corteza parietal & $9(53)$ \\
\hline Corteza temporal & $5(29,4)$ \\
Corteza occipital & $3(17,6)$ \\
Ínsula & $3(17,6)$ \\
Caudado & $14(82,4)$ \\
Putamen & $7(41,2)$ \\
Tálamo & $9(52,9)$ \\
\hline
\end{tabular}

RM: Resonancia magnética; DWI: Diffusion-weighted Imaging; FLAIR: fluid attenuated inversión recovery; ADC: Apparent Diffusion Coefficient.
Tabla 4. Principales hallazgos del electroencefalograma

\begin{tabular}{|c|c|}
\hline Hallazgo & n $(\%)$ \\
\hline Normal & $1 \quad(5,9)$ \\
\hline Actividad epileptiforme interictal focal & $4(23,5)$ \\
\hline Lentitud theta-delta generalizada aislada & $2(11,8)$ \\
\hline Patrón pseudoperiódico & $5(29,4)$ \\
\hline \multicolumn{2}{|l|}{ Status epiléptico } \\
\hline Generalizado & $2(11,8)$ \\
\hline Focal & $1 \quad(5,9)$ \\
\hline Total & $3(17,6)$ \\
\hline PLEDs & $2(11,8)$ \\
\hline
\end{tabular}

En todos los casos anormales, se encontró lentitud thetadelta generalizada como ritmo de base. PLEDs: periodic lateralized epileptiform discharges.

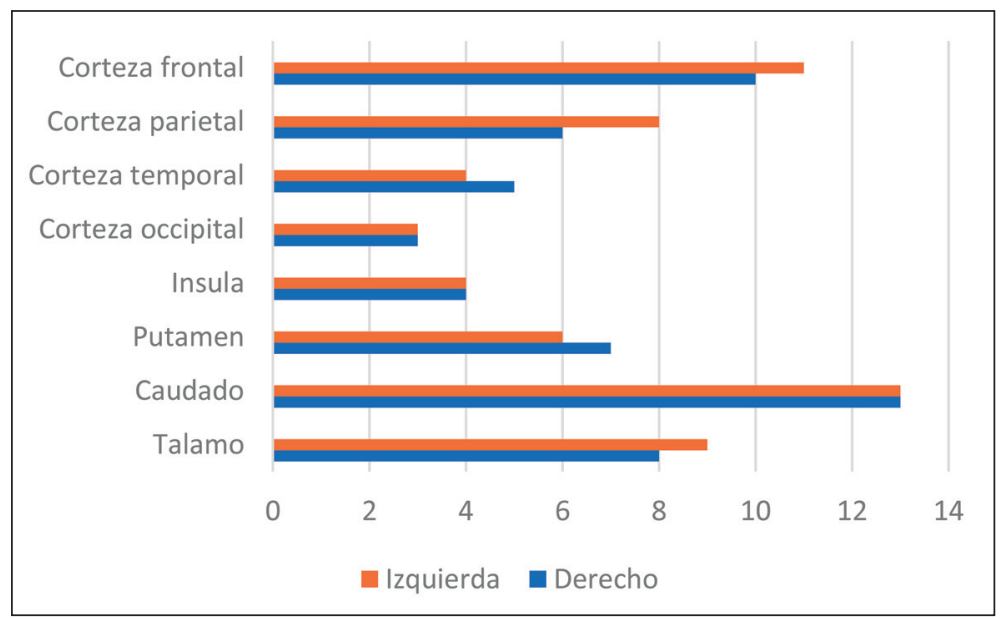

Figura 1. Lateralidad de la afectación de las diferentes áreas comprometidas en resonancia magnética.

\section{Electroencefalograma}

La mediana de tiempo entre el inicio de los síntomas y la realización del examen fue de 2 meses (Q1:2, Q3:11). El 94,1\% de los pacientes tuvo un EEG alterado. El 64,7\% de los pacientes se realizó el EEG dentro de los primeros 3 meses de la enfermedad. Los principales hallazgos patológicos se esquematizan en la Tabla 4. Todos los pacientes con EEG alterado presentaron una lentitud theta-delta generalizada como ritmo de base, siendo este un hallazgo exclusivo en $11,8 \%$. El patrón pseudoperiódico se encontró en 5 pa- cientes, de los cuales 4 presentaban una evolución de 3 meses o menos.

\section{Discusión}

El HBLT es un centro terciario, ubicado en el sector sur de la ciudad de Santiago de Chile y que tiene una población inscrita de aproximadamente 1,2 millones de personas, siendo la población realmente atendida de aproximadamente 1 millón ${ }^{12}$. Cuenta con un servicio de neurología hospitalaria, 
una unidad de electroencefalograma y un servicio de radiología, lo que permite realizar en todos los casos un estudio de imagen y electroencefalográfico adecuado en pacientes con demencia de curso rápidamente progresivo. En nuestro estudio, estimamos una incidencia acumulada de ECJ de 4,7 casos al año por millón de habitantes, lo que es concordante con el mayor número de casos reportados en nuestro país ${ }^{7,8}$.

La incidencia acumulada estimada en este estudio merece algunas consideraciones. Si bien la población atendida en nuestro hospital es de 1 millón de personas, hay que considerar que la ECJ se presenta principalmente en personas sobre 60 años, aunque se han reportado casos en paciente joven, hasta los 25-30 años ${ }^{2,13}$, por lo tanto, la población susceptible de desarrollar la enfermedad, considerando el número de personas sobre 20 años que se atienden en el HBLT corresponde a 740.000 personas $^{12}$. Por otra parte, si bien el HBLT es un centro terciario donde se derivan pacientes neurológicos desde centros públicos de menor complejidad del área sur de Santiago, esta serie de casos no considera pacientes hospitalizados en otros centros (públicos o privados) dentro del cálculo, por lo cual, este número podría ser aun mayor.

La distribución etaria reportada en series nacionales e internacionales muestra una mayor incidencia sobre los 60 años, una leve predominancia en mujeres $(1,4: 1)$ y un promedio de sobrevida en torno a los 6 a 7 meses para todos los subtipos genéticos ${ }^{4}$. Nuestra serie muestra aspectos similares, ya que se encontró una discreta preponderancia en mujeres y aproximadamente dos tercios de los casos se presentaron sobre los 60 años. La mediana del tiempo de sobrevida fue levemente menor a lo reportado en la literatura. Cabe destacar la presencia de dos pacientes con inicio de los síntomas antes de los 50 años, quienes no tenían antecedentes familiares de la enfermedad.

Como se mencionó previamente, la forma más frecuente de ECJ es la esporádica, seguida de la forma familiar ${ }^{6}$, siendo esta última mucho más frecuente en nuestro país, en comparación a lo descrito internacionalmente, con alrededor de un tercio de los casos $^{7,14}$. En nuestra serie, el 23,5\% tenía el antecedente de un pariente afectado en primer o segundo grado, lo cual se condice con lo anteriormente expuesto. Esto tiene importantes repercusiones en la familia, en cuanto a cuestio- namientos sobre la posibilidad de desarrollar la enfermedad.

La ECJ se caracteriza por tener una presentación clínica heterogénea donde el síntoma cardinal es la demencia de curso rápidamente progresivo, asociado a síntomas constitucionales inespecíficos (compromiso del estado general, baja de peso, alteraciones del sueño), síntomas neuropsiquiátricos (depresión, irritabilidad, agresividad, apatía, cambios de personalidad) y síntomas neurológicos variados que revelan el depósito de la proteína priónica anormal en diferentes localizaciones del encéfalo $^{4,15}$. Los hallazgos clínicos neurológicos que más comúnmente hacen sospechar esta enfermedad son las alteraciones de funciones cerebrales superiores, las mioclonías y la ataxia ${ }^{16}$, los cuales son los síndromes más frecuentemente encontrados en nuestra serie al momento de la consulta. Esta presentación clínica asociada a la edad de presentación y tiempo promedio de sobrevida podrían hacer presumir que la mayoría de los pacientes de nuestra serie corresponderían a la variante $\mathrm{MM1/MV1}$, la más frecuente dentro de los genotipos descritos $(65 \%)^{2,4}$. Otro aspecto relevante es la presencia de síndrome convulsivo en casi un tercio de los casos, lo cual fue superior a la mayoría de las series, en las cuales se reporta en $10-15 \%{ }^{17}$. Además, casi un quinto de los pacientes presentó un SEC al momento del ingreso. Si bien, en la literatura actual no se considera una presentación clínica habitual, se han descrito casos de SEC generalizado, focal ${ }^{17,18}$ y status epiléptico no convulsivo (SENC) ${ }^{19,20}$. Por lo tanto, en pacientes añosos con status epiléptico sin etiología clara, se debe considerar la ECJ dado su pronóstico.

La sensibilidad y especificidad reportados para la RM son de $83-95 \%^{21}$ y $92-96 \%{ }^{4,22,23}$, respectivamente, lo cual lo convierte en un examen muy útil para el diagnóstico. En nuestra serie, la RM se encontró alterada en todos los casos, lo cual se condice con lo señalado. Los hallazgos imagenológicos principales fueron la hiperintensidad del núcleo caudado, seguido de corteza frontal, parietal, tálamo y putamen. En cuanto a la lateralidad, se observó, en general, un compromiso simétrico. Cabe mencionar que el compromiso de la corteza frontal imagenológicamente fue de igual porcentaje al compromiso clínico y que la afectación occipital no fue preponderante, lo cual coincide con el bajo porcentaje de pacientes con manifestaciones clínicas visuales en nuestra 
serie. Si bien los criterios imagenológicos para el diagnóstico de ECJ excluyen las lesiones aisladas en corteza frontal por el aumento de falsos positivos en la secuencia de $\mathrm{DWI}^{10}$, en nuestro estudio esta región sí fue contemplada, ya que en todos los casos se acompañó de alteraciones en otras localizaciones.

El estudio electroencefalográfico es otro elemento que apoya el diagnóstico, aunque tiene como limitación su baja sensibilidad $(66 \%)^{21}$. El hallazgo típico corresponde a la actividad pseudoperiódica, que también puede presentarse en enfermedades con manifestaciones clínicas similares a ECJ (encefalitis autoinmune, encefalopatía hepática, intoxicación por litio, linfoma intracerebral) ${ }^{24}$. Este patrón suele ser tardío (generalmente después de los 3 meses) y se da principalmente en las formas MM1/MV1 (hasta $80 \%)^{2}$, siendo infrecuente en otros genotipos, lo cual explicaría en parte su sensibilidad ${ }^{25}$. En nuestra serie, encontramos este patrón en aproximadamente un tercio de los casos, pero al contrario de lo descrito, 4 de los 5 pacientes que presentaron esta alteración lo hicieron en los primeros 3 meses de la enfermedad, lo cual puede ser atribuido al bajo tamaño de la muestra. Solo un paciente presentó un EEG normal, el cual fue realizado tardíamente (12 meses) pudiendo tratarse de una forma distinta a MV1/MM1, ya que además la sobrevida fue de 13 meses. El hallazgo más común fue la presencia de un enlentecimiento generalizado como ritmo de base, que da cuenta de una afectación difusa o multifocal cerebral y que suele estar presente desde etapas tempranas ${ }^{26}$. Por último, es de gran interés la presencia de status epiléptico en casi un quinto de los casos, dentro de los cuales se encontraron dos status generalizados y uno focal. En nuestra serie no se encontraron pacientes con status epiléptico no convulsivo, pero existen múltiples reportes de su ocurrencia en esta enfermedad y muchas veces, este puede coexistir con la actividad pseudoperiódica, pudiendo incluso enmascararla ${ }^{16,20,27}$.

El principal diagnóstico diferencial de ECJ a considerar actualmente es la encefalitis autoinmune (asociada a neoplasia o no) y durante casi todo el período de esta revisión, el panel de anticuerpos para su estudio no estaba disponible en Chile, por lo cual no se dispuso del examen en la gran mayoría de los casos.

Respecto al tratamiento, no existe terapia cu- rativa, por lo que se basa en manejo sintomático (manifestaciones neuropsiquiátricas, infecciones, convulsiones), prevención de complicaciones asociadas a la postración, cuidados de fin de vida y acompañamiento a las familias.

\section{Conclusión}

La ECJ es la enfermedad priónica más frecuente, no obstante, es una entidad poco habitual, con una tasa de incidencia llamativamente más alta en nuestro país. Dado su amplio espectro de manifestaciones sistémicas, psiquiátricas y neurológicas, requiere un alto índice de sospecha luego de realizar el diagnóstico diferencial con otras enfermedades potencialmente reversibles, buscando apoyo en los exámenes paraclínicos que sugieran su probable existencia. Evitar el retraso de su diagnóstico es muy importante, ya que nos permite informar a la familia sobre el pronóstico funcional y vital del paciente, que indeclinablemente lleva hacia la muerte en un período corto de tiempo.

\section{Referencias}

1. Pan KM, Baldwin M, Nguyen J, Gasset M, Serban A, Groth D, et al. Conversion of alpha-helices into beta-sheets features in the formation of the scrapie prion proteins. Proc Natl Acad Sci USA 1993; 90: 10962-6.

2. Manix M, Kalakoti P, Henry M, Thakur J, Menger R, Guthikonda B, et al. Creutzfeldt-Jakob disease: updated diagnostic criteria, treatment algorithm, and the utility of brain biopsy. Neurosurg Focus. 2015; 39 (5): E2.

3. Cartier L. Enfermedad por priones, encefalopatía espongiforme humana y enfermedad de Creutzfeldt-Jakob. Rev Med Chile 2019; 147: 1176-83.

4. Zerr I, Parchi P. Sporadic Creutzfeldt-Jakob disease. Handbook of Clinical Neurology, Human Prion Diseases. M. Pocchiari and J. Manson, Editors 2018; Vol. 153 (3rd series).

5. Parchi P, Giese A, Capellari S, Brown P, Schulz-Schaeffer W, Windl O, et al. Classification of sporadic Creutzfeldt-Jakob disease based on molecular and phenotypic analysis of 300 subjects. Ann Neurol. 1999;46(2):224-33.

6. Uttley L, Carroll C, Wong R, Hilton DA, Stevenson M. Creutzfeldt-Jakob disease: a systematic review of global incidence, prevalence, infectivity, and incubation. Lancet Infect Dis. 2020; 20 (1): e2-e10. 
7. Cartier L, Quiroz G, Leiva M, Vergara C. Identificación clínica y patológica de las diversas formas de la enfermedad de Creutzfeldt Jakob en Chile. Rev Med Chile 2012; 140: 161-8.

8. Ramírez M, Gallardo A, Vidal A, Cornejo S, Ramírez D, Medinas D, et al. Desafíos en el diagnóstico de enfermedad de Creutzfeldt-Jakob. Caso clínico. Rev Med Chile 2016; 144: 806-11.

9. Bongianni M, Orrù C, Groveman BR, Sacchetto L, Fiorini M, Tonoli G, et al. Diagnosis of Human Prion Disease Using Real-Time Quaking-Induced Conversion Testing of Olfactory Mucosa and Cerebrospinal Fluid Samples. JAMA Neurol. 2017; 74 (2): 155-62.

10. Zerr I, Kallenberg K, Summers DM, Romero C, Taratuto A, Heinemann U, et al. Updated clinical diagnostic criteria for sporadic Creutzfeldt-Jakob disease. Brain. 2009 Oct; 132(Pt 10): 2659-68.

11. Mackenzie G and Will R. Creutzfeldt-Jakob disease: recent developments [version 1; referees: 2 approved] F1000 Research 2017; 6 (F1000 Faculty Rev): 205.

12. Cuenta Pública Hospital Barros Luco 2019. Disponible en http://www.hospitalbarrosluco.cl/wp-content/ uploads/2020/05/Cuenta-Publica-2019-HBLT.pdf

13. Boëlle P, Cesbron J, Valleron A. Epidemiological evidence of higher susceptibility to $\mathrm{VCJD}$ in the young. BMC Infect Dis. 2004; 4: 26.

14. Cartier L, Fernández J, Ramírez E. [Genetic markers in four Chilean families with familial Creutzfeldt-Jakob disease]. Rev Med Chile 2006; 134: 1116-22.

15. Torres Herrán GE, Ortega Heredia AD, Martínez B, Serrano-Dueñas M, Ortiz MA, Barrera RA, et al. Case series of Creutzfeldt-Jakob disease in a third-level hospital in Quito [published correction appears in BMC Neurol. 2018 Jun 12; 18 (1): 84]. BMC Neurol. 2018; 18 (1): 55. Published 2018 Apr 27.

16. Katsikaki G, Dagklis I, Angelopoulos P, Ntantos D, Prevezianou A, Bostantjopoulou S. Atypical and Early Symptoms of sporadic Creutzfeldt-Jakob Disease: Case series and review of the literature. Int J Neurosci. 2021; 131(9): 927-38.

17. Neufeld M, Talianski-aronov A, Soffer D, Korczyn A. Generalized convulsive status epilepticus in Creutzfeldt-
Jakob disease. Seizure 2003; 12: 403-5.

18. Taskiran A, Tezer FI, Saygi S. Epilepsia partialis continua as the presenting symptom in probable sporadic Creutzfeldt-Jakob disease. Epileptic Disord. 2011; 13 (1): 82-7.

19. Aiguabella M, Falip M, Veciana M, Bruna J, Palasí A, Corral L, et al. Refractory nonconvulsive status epilepticus in Creutzfeldt-Jakob disease. Epileptic Disord. 2010; 12 (3): 239-42.

20. Marquetand J, Knake S, Strzelczyk A, Steinhoff B, Lerche $\mathrm{H}$, Synofzik M, et al. Periodic EEG patterns in sporadic Creutzfeld-Jakob-Disease can be benzodiazepine responsive and interpreted as non-convulsive status epilepticus. Seizure 2017; 53: 47-50.

21. Warden D, Dennison J, Limback J, Shroff SM, Messina SA, et al. Imaging Manifestations of Creutzfeldt-Jakob Disease and case series. Cureus. 2018; 10 (12): e3725.

22. Fragoso DC, Gonçalves Filho AL, Pacheco FT, Barros BR, Aguiar Littig I, Nunes RH, et al. Imaging of Creutzfeldt-Jakob Disease: Imaging Patterns and Their Differential Diagnosis. Radiographics. 2017 Jan-Feb; 37 (1): 234-57.

23. Eisenmenger L, Porter M, Carswell C, Thompson A, Mead S, Rudge P, et al. Evolution of Diffusion-Weighted Magnetic Resonance Imaging Signal Abnormality in Sporadic Creutzfeldt-Jakob Disease, With Histopathological Correlation Jama Neurol. 2016; 73 (1): 76-84.

24. Steinhoff BJ, Zerr I, Glatting M, Schulz-Schaeffer W, Poser S, Kretzschmar HA. Diagnostic value of periodic complexes in Creutzfeldt-Jakob disease. Ann Neurol. 2004; 56 (5): 702-8.

25. Shin J-W, Yim B, Oh SH, Kim NK, Lee Sk, Kim O-J. Redefining Periodic Patterns on Electroencephalograms of Patients with Sporadic Creutzfeldt-Jakob Disease.Clin Neurphysiol 2017; 128 (5): 756-62.

26. Wieser HG, Schindler K, Zumsteg D. EEG in Creutzfeldt-Jakob disease. Clin Neurophysiol. 2006; 117 (5): 935-51.

27. Rakitin A, Vibo R, Veikat V, Ounapuu A, Liigant A, Haldre S. Case report. Creutzfeldt-Jakob disease presenting as nonconvulsive status epilepticus. Hindawi Case Reports in Neurological Medicine 2018; 4. Article ID 3092018. 